\title{
Exact BER of Rectangular-Constellation Quadrature Amplitude Modulation Subjected to Asynchronous Co-Channel Interference and Nakagami- $m$ Fading
}

\author{
Xiang Liu and Lajos Hanzo \\ School of Electronics and Computer Science, University of Southampton, SO17 1BJ, UK \\ lh@ecs.soton.ac.uk, www-mobile.ecs.soton.ac.uk
}

\begin{abstract}
Quadrature Amplitude Modulation (QAM) is a bandwidth-efficient transmission technique. The exact average Bit Error Ratio (BER) of the maximum-minimum-distance rectangular QAM (R-QAM) constellation is studied in the context of asynchronous Co-Channel Interference (CCI) and Nakagami- $m$ fading. A new formula is derived for the Characteristic Function (CF) of the CCI, which requires no knowledge of the CCI distribution. The numerical results obtained from our exact BER expression are verified by our simulation results and are also compared to those of the Gaussian Approximation (GA).
\end{abstract}

\section{INTRODUCTION}

The family of Quadrature Amplitude Modulation (QAM) [1] schemes has found its way into virtually all recent wireless standards, including the third-generation (3G) High-Speed Downlink Packet Access (HSDPA), the 802.11 Wireless Local Area Network (WLAN) family, as well as the Digital Audio Broadcast (DAB) and Video Broadcast (DVB) systems. The maximum-minimum distance rectangular QAM (R-QAM) constellation is popular, since it achieves the best BER in uncoded Gaussian scenarios.

The Symbol Error Rate (SER) performance of R-QAM has been studied using various exact computation techniques in [2]-[6]. By contrast, the novel contribution of this paper is that we evaluate the BER performance of R-QAM, when additionally the Co-Channel Interference (CCI) is taken into account. Conventionally, the R-QAM BER has been estimated by using various approximations or bounds [2], [7]. However, using exact BER calculation is still desirable for verifying the accuracy of various approximation and bounding techniques. The exact BER expressions derived for 16-QAM and 64QAM constellations were provided in [1]. A general recursive algorithm devised for the exact BER computation of Square QAM (S-QAM) was presented in [8], while an exact and general closed-form BER expression of R-QAM ${ }^{1}$ was derived for arbitrary constellation sizes in [9]. Most of these results were obtained for Additive White Gaussian Noise (AWGN)

The financial support of the EPSRC, UK and the EU in the framework of the NEWCOM, NEXWAY and PHOENIX projects is gratefully acknowledged.

${ }^{1}$ The generic class of R-QAM contains both the specific subclass of squareshaped S-QAM constellations, as well as non-square constellations. channels. The AWGN result of [9] was later extended to Nakagami- $m$ [10], [11] and Ricean [12] fading channels. A signal-space partitioning method was proposed for calculating the exact SER/BER of arbitrary two-dimensional signaling in the context of various fading channels in [13].

The exact QAM BER calculation becomes even more challenging, when the CCI is taken into account. Conventionally, the CCI is assumed to be Gaussian distributed for the sake of computational simplicity [14]. However, the Gaussian Approximation (GA) is accurate only, when we have a large number of interferers owning to the Central Limit Theorem (CLT) [15]. Moreover, to the best of the authors' knowledge, the exact and general BER expression of R-QAM systems corrupted by $\mathrm{CCI}$ has not been derived. In the existing literature, most exact BER analyses procedures were performed for BPSK [16]-[25] and QPSK [24]-[27] systems.

Hence, again, the contribution of this paper is that we derive an exact and general BER expression for general R-QAM systems corrupted by both asynchronous CCI and Nakagami$m$ fading, while dispensing with the Gaussian distributed CCI assumption. This paper is organized as follows. In Section II a general R-QAM system subject to asynchronous CCI and Nakagami- $m$ fading is described. Its exact BER performance is investigated based on the Characteristic Function (CF) approach in Section III. Our numerical results are presented in Section IV, where we verify the accuracy of our exact BER expression and demonstrate the limited accuracy of the GA method. Finally, we conclude this paper in Section V.

\section{SySTEM MOdeL}

The R-QAM signal consists of two independent amplitudemodulated signals and can be expressed as [10]:

$$
s(t)=d^{I} b^{I}(t) \cos \left(\omega_{c} t+\theta\right)+d^{Q} b^{Q}(t) \sin \left(\omega_{c} t+\theta\right),
$$

where $\omega_{c}$ and $\theta$ are the common carrier frequency and the carrier phase shift. As illustrated in [9], $2 d^{I}$ and $2 d^{Q}$ are the minimum distance between signal constellation points along the in-phase and quadrature-phase axes, respectively. Note that $d^{I}$ and $d^{Q}$ are not necessarily equal in the general rectangular 
QAM constellation. The in-phase and quadrature-phase data signals, $b^{I}(t)$ and $b^{Q}(t)$, are given by:

$$
\begin{aligned}
& b^{I}(t)=\sum_{n=-\infty}^{\infty} b_{n}^{I} p_{T_{s}}\left(t-n T_{s}\right), \\
& b^{Q}(t)=\sum_{n=-\infty}^{\infty} b_{n}^{Q} p_{T_{s}}\left(t-n T_{s}\right),
\end{aligned}
$$

where $\left\{b_{n}^{I}\right\}_{n=-\infty}^{\infty}$ and $\left\{b_{n}^{Q}\right\}_{n=-\infty}^{\infty}$ are the in-phase and quadrature-phase data symbols, respectively. The symbol duration is denoted as $T_{s}$ and $p_{T}(t)$ is the rectangular pulse having a duration of $T$, i.e. we have

$$
p_{T}(t)=\left\{\begin{array}{l}
1, t \in[0, T) \\
0, \text { otherwise. }
\end{array}\right.
$$

In the $M$-ary R-QAM scheme, where we have $M=$ $M^{I} \times M^{Q}, \log _{2} M^{I}$ and $\log _{2} M^{Q}$ bits are Gray encoded and mapped onto the in-phase and quadrature-phase components [1], [9], respectively. Hence, the in-phase and quadrature data symbols, $b_{n}^{I}$ and $b_{n}^{Q}$, are selected from the set of $\mathcal{A}^{I}=$ $\left\{ \pm 1, \pm 3, \ldots, \pm\left(M^{I}-1\right)\right\}$ and $\mathcal{A}^{Q}=\left\{ \pm 1, \pm 3, \ldots, \pm\left(M^{Q}-\right.\right.$ 1)\}, respectively.

We consider a general R-QAM system subjected to $K$ asynchronous co-channel interferers. The received signal $r(t)$ subjected to fading may be written as:

$$
\begin{aligned}
r(t)= & \sum_{k=0}^{K} h_{k}\left\{d_{k}^{I} b_{k}^{I}\left(t-\tau_{k}\right) \cos \left[\omega_{c}\left(t-\tau_{k}\right)+\theta_{k}+\varphi_{k}\right]\right. \\
& \left.+d_{k}^{Q} b_{k}^{Q}\left(t-\tau_{k}\right) \sin \left[\omega_{c}\left(t-\tau_{k}\right)+\theta_{k}+\varphi_{k}\right]\right\}+\eta(t),
\end{aligned}
$$

where the fading amplitude $h_{k}$ obeys the Nakagami- $m$ distribution having parameters $\left\{m_{k}, \Omega_{k}\right\}$ [28], the fading phase $\varphi_{k}$ is typically assumed to be uniformly distributed over $[0,2 \pi)$ [28], the time delay $\tau_{k}$ of the $k$ th user is uniformly distributed over $\left[0, T_{s}\right)$, and the Additive White Gaussian Noise (AWGN) $\eta(t)$ has a double-sided power spectral density of $N_{0} / 2$.

Without loss of generality, we assume that the 0th user is the desired one. In the case of coherent demodulation as well as perfect channel estimation, the in-phase and quadrature-phase decision statistics, $Z^{I}$ and $Z^{Q}$, are given by:

$$
Z^{I}=d_{0}^{I} h_{0} b_{0,0}^{I}+\sum_{k=1}^{K} h_{k}\left(X_{k}^{I} \cos \Delta_{k}+X_{k}^{Q} \sin \Delta_{k}\right)+\eta^{I},(6)
$$

$Z^{Q}=d_{0}^{Q} h_{0} b_{0,0}^{Q}+\sum_{k=1}^{K} h_{k}\left(X_{k}^{Q} \cos \Delta_{k}-X_{k}^{I} \sin \Delta_{k}\right)+\eta^{Q}$ (7)

where the phase shift difference $\Delta_{k}=-\omega_{c}\left(\tau_{k}-\tau_{0}\right)+\left(\theta_{k}-\right.$ $\left.\theta_{0}\right)+\left(\varphi_{k}-\varphi_{0}\right)$ between the $k$ th interferer and the desired user is uniformly distributed over $[0,2 \pi)$. The noise components $\eta^{I}$ and $\eta^{Q}$ can be shown to be zero-mean Gaussian distributed random variables, both having a variance of $N_{0} / T_{s}$. The random variables $X_{k}^{I}$ and $X_{k}^{Q}$ are defined as:

$$
\begin{aligned}
X_{k}^{I} & =d_{k}^{I}\left[b_{k,-1}^{I} \nu_{k}+b_{k, 0}^{I}\left(1-\nu_{k}\right)\right], \\
X_{k}^{Q} & =d_{k}^{Q}\left[b_{k,-1}^{Q} \nu_{k}+b_{k, 0}^{Q}\left(1-\nu_{k}\right)\right],
\end{aligned}
$$

where $\nu_{k}=\tau_{k} / T_{s}$ is the time delay of the $k$ th interferer normalized by the symbol duration.

\section{BER ANALYSIS}

Let us now continue by analyzing the error probability of the in-phase component based on the CF approach. The error probability of the quadrature component may be derived in the same way.

Upon exploiting the results of [29], we have the CF of the in-phase CCI $I_{k}^{I}=h_{k}\left(X_{k}^{I} \cos \Delta_{k}+X_{k}^{Q} \sin \Delta_{k}\right)$ conditioned on $X_{k}^{I}$ and $X_{k}^{Q}$ in the following form:

$\Phi_{I_{k}^{I} \mid X_{k}^{I}, X_{k}^{Q}}(\omega)={ }_{1} \mathbb{F}_{1}\left(m_{k} ; 1 ;-\frac{\Omega_{k}}{4 m_{k}}\left[\left(X_{k}^{I}\right)^{2}+\left(X_{k}^{Q}\right)^{2}\right] \omega^{2}\right)$

where ${ }_{1} \mathbb{F}_{1}(\alpha ; \beta ; x)$ is the confluent hypergeometric function [30]. Upon averaging $\Phi_{I_{k}^{I} \mid X_{k}^{I}, X_{k}^{Q}}(\omega)$ over the $k$ th interferer's data symbols $b_{k,-1}^{I}, b_{k,-1}^{Q}, b_{k, 0}^{I}, b_{k, 0}^{Q}$ and the time delay $\tau_{k}$, we obtain the CF of $I_{k}^{I}, \Phi_{I_{k}^{I}}(\omega)$, as follows:

$$
\Phi_{I_{k}^{I}}(\omega)=\frac{1}{M_{k}^{2}} \sum_{b_{k,-1}^{I}, b_{k, 0}^{I} \in \mathcal{A}_{k}^{I}} \sum_{b_{k,-1}^{Q}, b_{k, 0}^{Q} \in \mathcal{A}_{k}^{Q}} \Phi_{I_{k}^{I} \mid \lambda_{0}, \lambda_{1}, \lambda_{2}}(\omega),
$$

where the coefficients $\lambda_{0}, \lambda_{1}$ and $\lambda_{2}$ are defined as:

$\lambda_{0}=\left(d_{k}^{I}\right)^{2}\left(b_{k, 0}^{I}\right)^{2}+\left(d_{k}^{Q}\right)^{2}\left(b_{k, 0}^{Q}\right)^{2}$,

$\lambda_{1}=\left(d_{k}^{I}\right)^{2} b_{k, 0}^{I}\left(b_{k,-1}^{I}-b_{k, 0}^{I}\right)+\left(d_{k}^{Q}\right)^{2} b_{k, 0}^{Q}\left(b_{k,-1}^{Q}-b_{k, 0}^{Q}\right)$,

$\lambda_{2}=\left(d_{k}^{I}\right)^{2}\left(b_{k,-1}^{I}-b_{k, 0}^{I}\right)^{2}+\left(d_{k}^{Q}\right)^{2}\left(b_{k,-1}^{Q}-b_{k, 0}^{Q}\right)^{2}$.

The conditional CF, $\Phi_{I_{k}^{I} \mid \lambda_{0}, \lambda_{1}, \lambda_{2}}(\omega)$, may be shown to be given by Equation 15 seen at the top of the next page. When we have $M_{k}=2$, i.e. the $k$ th interferer adopts BPSK modulation and experiences Nakagami- $m$ fading, Equation 15 reduces to Equations 8 and 9 of [25]. By contrast, when $M_{k}=4, d_{k}^{I}=d_{k}^{Q}$ and $m=1$, i.e. the $k$ th interferer adopts QPSK modulation and experiences Rayleigh fading, Equation 15 reduces to Equations 17, 19 and 21 of [25].

The in-phase CCI $I_{k}^{I}$ imposed by the different interferers is mutually independent. Upon defining the total in-phase interference plus noise term as $\xi^{I}=\sum_{k=1}^{K} I_{k}^{I}+\eta^{I}$, it transpires that both its $\operatorname{PDF} f_{\xi^{I}}(x)$ and its $\operatorname{CF} \Phi_{\xi^{I}}(\omega)=\Phi_{\eta^{I}}(\omega) \prod_{k=1}^{K} \Phi_{I_{k}^{I}}(\omega)$ are even. Hence the Cumulative Distribution Function (CDF) $\mathrm{F}_{\xi^{I}}(x)$ of the total in-phase interference plus noise can be shown to be:

$$
\mathrm{F}_{\xi^{I}}(x)=\frac{1}{2}+\frac{1}{\pi} \int_{0}^{\infty} \frac{\sin (\omega x)}{\omega} \Phi_{\xi^{I}}(\omega) \mathrm{d} \omega .
$$

Extending the AWGN result of [9] to the scenarios encountered in presence of interference plus noise, the conditional error probability of the $u$ th bit of the in-phase component, 


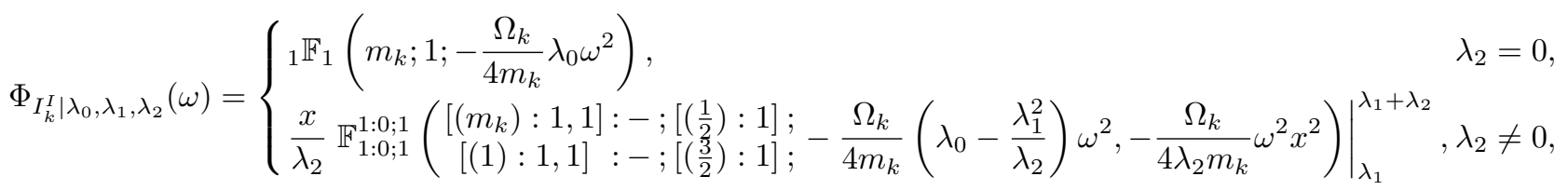

where $\left.\mathbb{F}_{C: D^{(1)} ; \ldots ; D^{(n)}}^{A: B^{(1)} \ldots ; B^{(n)}}\left(\left[(a): \theta^{(1)}, \ldots, \theta^{(n)}\right]:\left[\left(b^{(1)}\right): \phi^{(1)}\right] ; \ldots ;\left[\left(b^{(n)}\right): \phi^{(n)}\right] ; \psi^{(1)}, \ldots, \psi^{(n)}\right]:\left[\left(d^{(1)}\right): \delta^{(1)}\right] ; \ldots ;\left[\left(d^{(n)}\right): \delta^{(n)}\right] ; x_{1}, \ldots, x_{n}\right)$ is the generalized Lauricella function of $n$ variables defined as Equations $21-23$ of [31] and $\left.f(x)\right|_{x_{1}} ^{x_{2}}=f\left(x_{2}\right)-f\left(x_{1}\right)$.

$P_{b \mid h_{0}}^{I}(u)$, can be expressed as follows when $f_{\xi^{I}}(x)$ is even:

$$
\begin{aligned}
& P_{b \mid h_{0}}^{I}(u)=\frac{1}{M_{k}^{I}} \sum_{l=0}^{\left(1-2^{-u}\right) M_{k}^{I}-1}\left\{(-1)^{\left\lfloor\frac{l 2^{u-1}}{M_{k}^{I}}\right\rfloor}\right. \\
& \left.\times\left(2^{u-1}-\left\lfloor\frac{l 2^{u-1}}{M_{k}^{I}}+\frac{1}{2}\right\rfloor\right)\left[2\left(1-\mathrm{F}_{\xi^{I}}\left((2 l+1) d_{0}^{I} h_{0}\right)\right)\right]\right\},
\end{aligned}
$$

where $\lfloor x\rfloor$ denotes the largest integer no greater than $x$. Upon averaging $P_{b \mid h_{0}}^{I}(u)$ over $h_{0}$ and applying Parseval's theorem [32], we obtain the error probability $P_{b}^{I}(u)$ of the $u$ th bit of the in-phase component in the form of:

$$
\begin{aligned}
& P_{b}^{I}(u)=\frac{1}{2}-\frac{2}{\pi M_{k}^{I}} \int_{0}^{\infty} \frac{\Phi_{\xi^{I}}(\omega)}{\omega} \sum_{l=0}^{\left(1-2^{-u}\right) M_{k}^{I}-1}\left\{(-1)^{\left\lfloor\frac{l 2^{u-1}}{M_{k}^{I}}\right\rfloor}\right. \\
& \left.\times\left(2^{u-1}-\left\lfloor\frac{l 2^{u-1}}{M_{k}^{I}}+\frac{1}{2}\right\rfloor\right) \Im\left\{\Phi_{h_{0}}\left[(2 l+1) d_{0}^{I} \omega\right]\right\}\right\} \mathrm{d} \omega,
\end{aligned}
$$

where $\Im\left\{\Phi_{h_{0}}(\omega)\right\}$ is the imaginary part of the CF of the desired user's fading amplitude, $h_{0}$, which is given by Table II of [32].

Following the same approach, we may derive the error probability $P_{b}^{Q}(u)$ of the $u$ th bit of the quadrature-phase component in the form of:

$$
\begin{aligned}
& P_{b}^{Q}(u)=\frac{1}{2}-\frac{2}{\pi M_{k}^{Q}} \int_{0}^{\infty} \frac{\Phi_{\xi^{Q}}(\omega)}{\omega} \sum_{l=0}^{\left(1-2^{-u}\right) M_{k}^{Q}-1}\left\{( - 1 ) \left\lfloor\frac{l 2^{u-1}}{M_{k}^{Q}}\right.\right. \\
& \left.\times\left(2^{u-1}-\left\lfloor\frac{l 2^{u-1}}{M_{k}^{Q}}+\frac{1}{2}\right\rfloor\right) \Im\left\{\Phi_{h_{0}}\left[(2 l+1) d_{0}^{Q} \omega\right]\right\}\right\} \mathrm{d} \omega .
\end{aligned}
$$

Finally, the average BER of $M$-ary general R-QAM can be obtained by averaging the error probabilities given by Equations 18 and 19 [9]:

$$
P_{b}=\frac{1}{\log _{2} M_{0}}\left(\sum_{u=1}^{\log _{2} M_{0}^{I}} P_{b}^{I}(u)+\sum_{u=1}^{\log _{2} M_{0}^{Q}} P_{b}^{Q}(u)\right) .
$$

When there is no interference, i.e. we have $K=0$, Equations 18 and 19 reduce to the single-user results of [10], [11]. As expected, when only BPSK or QPSK are considered, i.e. we have $d_{k}^{I}=d_{k}^{Q}$ and $M_{k}=2,4$ for all users, Equations 18 and 19 reduce to the results of [25].

\section{NUMERICAL RESULTS}

In this section, we will verify the accuracy of our exact BER expression provided in Section III and demonstrate the limited accuracy of the GA method by simulations.

Since the evaluation of the effects of CCI on the QAM BER is the main objective of our analysis, we assume that the effects of noise are negligible. We assume furthermore that the minimum distances between signal points of the inphase and quadrature-phase components are the same, i.e. we have $d_{k}^{I}=d_{k}^{Q}$, which is typical in QAM, although our analysis in Section III also applies to more general cases, where $d_{k}^{I}$ and $d_{k}^{Q}$ are not necessarily equal. Furthermore, the average interference power imposed by each interferer is common and they experience the same fading statistics as the desired signal, i.e. we have the same $m_{k}$ value for all users, $k=0,1, \ldots, K$. Nevertheless, our analysis attained in Section III applies to various general cases, where the average power of each interferer is different or where each user experiences different fading distributions. We define the per-bit Signal-to-Interference Ratio (SIR) as:

$$
\mathrm{SIR}=\frac{1}{\log _{2} M_{0}} \frac{\left[\left(d_{0}^{I}\right)^{2}+\left(d_{0}^{Q}\right)^{2}\right] \Omega_{0}}{\sum_{k=1}^{K}\left[\left(d_{k}^{I}\right)^{2}+\left(d_{k}^{Q}\right)^{2}\right] \Omega_{k}} .
$$

Figures 1, and 2 illustrate the average BER performance versus the per-bit SIR expressed in $\mathrm{dB}$ in the context of Nakagami- $m$ fading channels associated with the parameters of $m=5$ and $m=10$, respectively. We assume that the number of interferers is $K=6$ in the simulations of both figures. This is typical in the hexagonal cellular model of TDMA cellular networks, where each cell is surrounded by $K=6$ adjacent so-called first-tier interfering cells and usually only the interference from these $K=6$ adjacent cells is considered. As seen in both figures, the results calculated by our exact BER analysis and the simulation results match both for various constellation sizes and for various Nakagami- $m$ fading parameters. On the other hand, the GA slightly overestimates the average BER. As expected, when the per-bit SIR is high, the fading becomes less severe, i.e. the Nakagami- $m$ parameter increases, and the number of bits/symbol is low, the GA becomes less accurate.

Although there are six adjacent first-tier interfering cells, different interferers may have different levels of influence. It is typical that there are one or two dominant interferers. 
Nakagami-m Fading $(\mathrm{m}=5)$

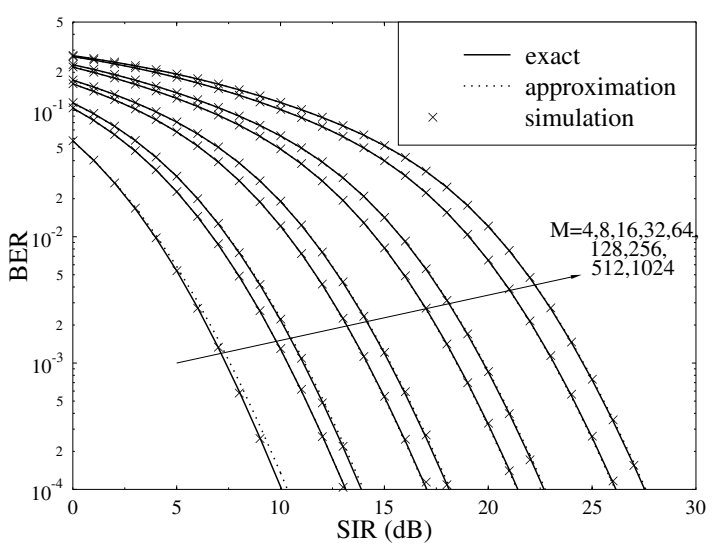

Fig. 1. BER versus the per-bit SIR in a R-QAM system subjected to asynchronous CCI and Nakagami- $m$ fading. All users have the same constellation size, i.e. $M_{k}=M$. The constellation size is $M=4,8,16,32$, $64,128,256,512$ and 1024 , respectively. The minimum distances between signal points of the in-phase and quadrature-phase components are the same, i.e. $d_{k}^{I}=d_{k}^{Q}$. The number of interferers is $K=6$. The average power of each interferer is common and they experience the same fading distribution as the desired signal, i.e. $m_{k}=5$. The background noise is ignored.

Nakagami-m Fading $(\mathrm{m}=10)$

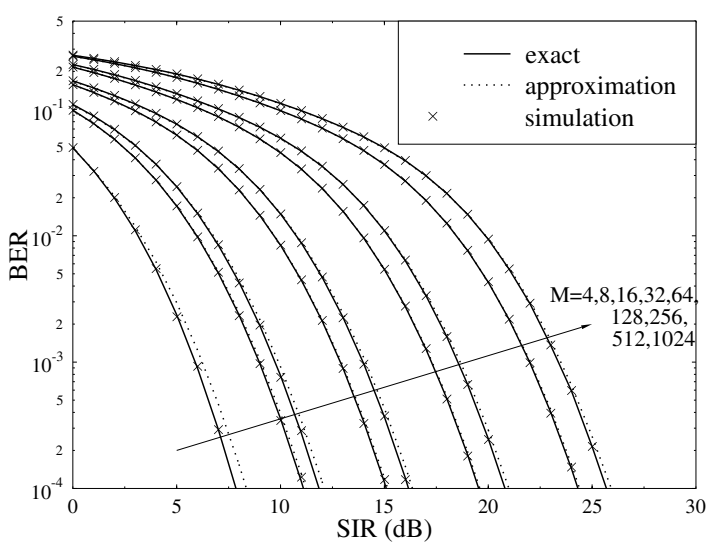

Fig. 2. BER versus the per-bit SIR in a R-QAM system subjected to asynchronous CCI and Nakagami- $m$ fading. All users have the same constellation size, i.e. $M_{k}=M$. The constellation size is $M=4,8,16,32$, $64,128,256,512$ and 1024 , respectively. The minimum distances between signal points of the in-phase and quadrature-phase components are the same, i.e. $d_{k}^{I}=d_{k}^{Q}$. The number of interferers is $K=6$. The average power of each interferer is common and they experience the same fading distribution as the desired signal, i.e. $m_{k}=10$. The background noise is ignored.

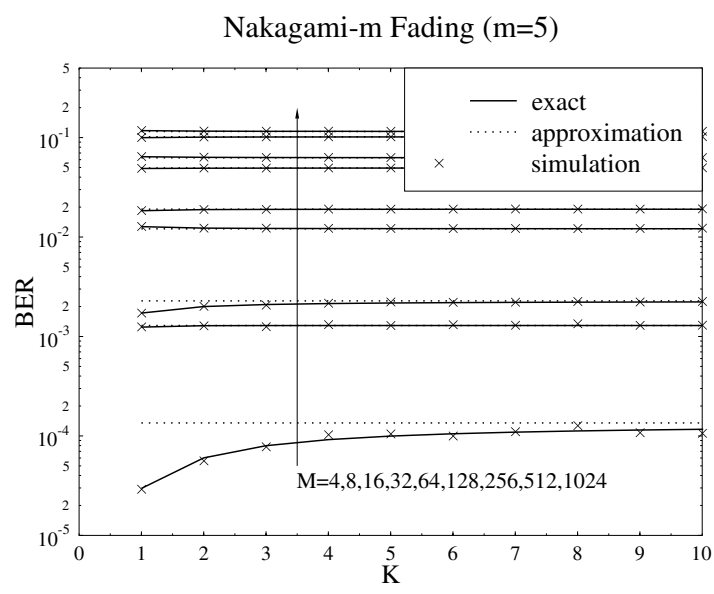

Fig. 3. BER versus the number of interferers in a R-QAM system subjected to asynchronous CCI and Nakagami- $m$ fading. All users have the same constellation size, i.e. $M_{k}=M$. The constellation size is $M=4,8,16,32$, $64,128,256,512$ and 1024 , respectively. The minimum distances between signal points of the in-phase and quadrature-phase components are the same, i.e. $d_{k}^{I}=d_{k}^{Q}$. The per-bit SIR is $10 \mathrm{~dB}$. The average power of each interferer is common and they experience the same fading distribution as the desired signal, i.e. $m_{k}=5$. The background noise is ignored.

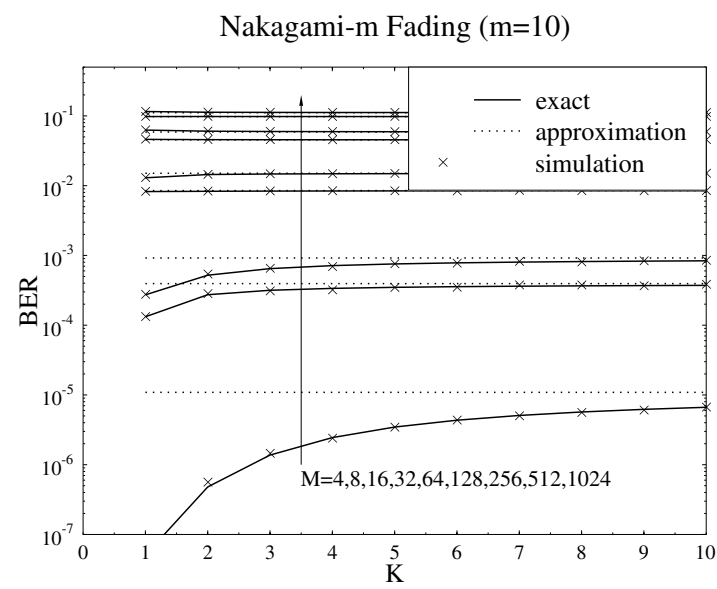

Fig. 4. BER versus the number of interferers in a R-QAM system subjected to asynchronous CCI and Nakagami- $m$ fading. All users have the same constellation size, i.e. $M_{k}=M$. The constellation size is $M=4,8,16,32$, $64,128,256,512$ and 1024 , respectively. The minimum distances between signal points of the in-phase and quadrature-phase components are the same, i.e. $d_{k}^{I}=d_{k}^{Q}$. The per-bit SIR is $10 \mathrm{~dB}$. The average power of each interferer is common and they experience the same fading distribution as the desired signal, i.e. $m_{k}=10$. The background noise is ignored. 
Figures 3 and 4 illustrate the average BER performance versus the number of interferers. For the sake of simplicity, we assume that the average power of all dominant interferers is the same and the influence of all non-dominant interferers is negligible. As we expected, the results obtained by our exact BER analysis and the simulation results match for various constellation sizes and various Nakagami- $m$ fading parameters. On the other hand, the GA over-estimates the average BER, especially when the constellation size is small, the fading becomes less severe and the number of interferers is small.

\section{CONCLUSION}

An exact and general BER expression has been derived for general R-QAM systems subjected to asynchronous CCI and Nakagami- $m$ fading, which requires only two single numerical integrations. A new closed-form formula was provided for the $\mathrm{CF}$ of the CCI with the aid of the generalized Lauricella function of $n$ variables [31]. Our simulation results verified the accuracy of our exact BER analysis for different constellation sizes and for various channel statistics. By contrast, the Gaussian model of CCI fails to accurately predict the QAM BER performance. Our future work may consider deriving similar formulae for both dispersive channels and for CDMA systems.

\section{REFERENCES}

[1] L. Hanzo, C. Wong, and M. Yee, Adaptive Wireless Tranceivers. John Wiley and Sons Ltd., 2002.

[2] J. G. Proakis, Digital Communications, 4th ed. McGraw-Hill Companies, Inc., 2001

[3] A. Annamalai, C. Tellambura, and V. K. Bhargava, "Exact Evaluation of Maximal-Ratio and Equal-Gain Diversity Receivers for $M$-ary QAM on Nakagami Fading Channels," IEEE Transactions on Communications, vol. 47, no. 9, pp. 1335-1344, September 1999.

[4] A. Annamalai and C. Tellambura, "Error Rates for Nakagami- $m$ Fading Multichannel Reception of Binary and $M$-ary Signals," IEEE Transactions on Communications, vol. 49, no. 1, pp. 58-68, January 2001.

[5] X. Dong, N. C. Beaulieu, and P. H. Wittke, "Error Probabilities of Two-Dimensional $M$-ary Signaling in Fading," IEEE Transactions on Communications, vol. 47, no. 3, pp. 352-355, March 1999.

[6] X. Dong and L. Xiao, "Symbol Error Probability of Two-Dimensional Signaling in Ricean Fading with Imperfect Channel Estimation," IEEE Transactions on Vehicular Technology, vol. 54, no. 2, pp. 538-549, March 2005.

[7] J. Lu, K. B. Letaief, J.-I. Chuang, and M. L. Liou, " $M$-PSK and $M$ QAM BER Computation Using Signal-Space Concepts," IEEE Transactions on Communications, vol. 47, no. 2, pp. 181-184, February 1999.

[8] L.-L. Yang and L. Hanzo, "A Recursive Algorithm for the Error Probability Evaluation of $M$-QAM," IEEE Communications Letters, vol. 4, no. 10, pp. 304-306, October 2000.

[9] K. Cho and D. Yoon, "On the General BER Expression of Oneand Two-Dimension Amplitude Modulations," IEEE Transactions on Communications, vol. 50, no. 7, pp. 1074-1080, July 2002.

[10] D. Yoon and K. Cho, "General Bit Error Probability of Rectangular Quadrature Amplitude Modulation," Electronics Letters, vol. 38, no. 3, pp. 131-133, January 2002.

[11] K. Cho, D. Yoon, W. Jeong, and M. Kavehrad, "BER Analysis of Arbitrary Rectangular QAM," in Thirty-Fifth Asilomar Conference on Signals, Systems and Computers, vol. 2, Pacific Grove, CA, 4-7 November 2001, pp. 1056-1059.

[12] J. S. Lim, K. Hyun, D. Yoon, and S. K. Park, "BER Performance of Rectangular QAM with MRC over Nakagami-n Fading Channels," IEICE Transactions on Communications, vol. E88-B, no. 4, pp. 16971701, April 2005
[13] L. Xiao and X. Dong, "The Exact Transition Probability and Bit Error Probability of Two-Dimensional Signaling," IEEE Transactions on Wireless Communications, vol. 4, no. 5, pp. 2600-2609, September 2005.

[14] L. Habbab, M.Kavehrad, and C. Sundberg, "ALOHA with Capture over Slow and Fast Fading Radio Channels with Coding and Diversity," IEEE Journal on Selected Areas in Commmunications, vol. 7, no. 1, pp. 79-88, January 1989.

[15] A. Papoulis, Probability, Random Variables, and Stochastic Processes, 3rd ed. McGraw-Hill, Inc., 1991.

[16] M. Chiani, "Performance of BPSK and GMSK with Multiple Cochannel Interferers," in IEEE International Symposium on Personal, Indoor and Mobile Radio Communications, vol. 3, Taipei, Taiwan, R.O.C., 15-18 October 1996, pp. 833-837.

[17] V. A. Aalo and J. Zhang, "On the Effect of Cochannel Interference on Average Error Rates in Nakagami-Fading Channels," IEEE Communications Letters, vol. 3, no. 5, pp. 136-138, May 1999.

[18] A. Shah, A. M. Haimovich, M. K. Simon, and M.-S. Alouini, "Exact Bit-Error Probability for Optimum Combining with a Rayleigh Fading Gaussian Cochannel Interferer," IEEE Transactions on Communications, vol. 48, no. 6, pp. 908-912, June 2000 .

[19] K. A. Hamdi, "Exact Probability of Error of BPSK Communication Links Subjected to Asynchronous Interference in Rayleigh Fading Environment," IEEE Transactions on Communications, vol. 50, no. 10, pp. 1577-1579, October 2002.

[20] J. Cheng and N. Beaulieu, "Error Rate of BPSK in Generalized Fading Channels with Co-Channel Interference," in IEEE Vehicular Technology Conference Spring, vol. 4, Birmingham, Al, USA, 6-9 May 2002, pp. $1786-1790$.

[21] N. Beaulieu and J. Cheng, "Precise Error-Rate Analysis of BandwidthEfficient BPSK in Nakagami Fading and Cochannel Interference," IEEE Transactions on Communications, vol. 52, no. 1, pp. 149-158, January 2004.

[22] K. Sivanesan and N. C. Beaulieu, "Exact BER Analysis of Bandlimited BPSK with EGC and SC Diversity in Cochannel Interference and Nakagami Fading," IEEE Communications Letters, vol. 8, no. 10, pp. 623-625, October 2004.

[23] Z. Du, J. Cheng, and N. C. Beaulieu, "BER Analysis of BPSK Signaling in Ricean-Faded Cochannel Interference," in IEEE Global Telecommunications Conference, St. Louis, Missouri, USA, 28 November - 2 December 2005, pp. 1211-1216.

[24] A. Giorgetti and M. Chiani, "Influence of Fading on the Gaussian Approximation for BPSK and QPSK with Asynchronous Cochannel Interference," IEEE Transactions on Wireless Communications, vol. 4, no. 2, pp. 384-389, March 2005.

[25] K. Sivanesan and N. C. Beaulieu, "Exact BER Analyses of Nakagami/Nakagami CCI BPSK and Nakagami/Rayleigh CCI QPSK Systems in Slow Fading," IEEE Communications Letters, vol. 8, no. 1, pp. 45-47, January 2004.

[26] N. C. Beaulieu and A. A. Abu-Dayya, "Bandwidth Efficient QPSK in Cochannel Interference and Fading," IEEE Transactions on Communications, vol. 43, no. 9, pp. 2464-2474, September 1995.

[27] J. Cheng, N. Beaulieu, and X. Zhang, "Precise BER Analysis of Dual-Channel Reception of QPSK in Nakagami Fading and Cochannel Interference," IEEE Communications Letters, vol. 9, no. 4, pp. 316-318, April 2005.

[28] M. Nakagami, "The $m$-Distribution - A General Formula of Intensity Distribution of Rapid Fading," in Statistical Methods in Radio Wave Propagation, W. C. Hoffman, Ed. London: Pergamon Press, 1960, pp. 3-36.

[29] Q. Shi and M. Latva-Aho, "Accurate Bit-Error Rate Evaluation for Synchronous MC-CDMA over Nakagami- $m$-Fading Channels Using Moment Generating Functions," IEEE Transactions on Wireless Communications, vol. 4, no. 2, pp. 422-433, March 2005.

[30] I. S. Gradshteyn and I. M. Ryzhik, Table of Integrals, Series, and Products, 6th ed., A. Jeffrey and D. Zwillinger, Eds. Academic Press, 2000.

[31] H. M. Srivastava and P. W. Karlsson, Multiple Gaussian Hypergeometric Series. Ellis Horwood, Ltd., 1985.

[32] A. Annamalai, C. Tellambura, and V. K. Bhargava, "Equal-Gain Diversity Receiver Performance in Wireless Channels," IEEE Transactions on Communications, vol. 48, no. 10, pp. 1732-1745, October 2000. 